\title{
Flame Detection using Image Processing Techniques
}

\author{
Punam Patel \\ M.tech (CSE) \\ MITS, Laxmangarh
}

\author{
Shamik Tiwari \\ Assistant professor \\ MITS, Laxmangarh
}

\begin{abstract}
Dynamic textures are common in natural scenes. Examples of dynamic textures in video include fire, smoke, trees in the wind, clouds, sky, ocean waves etc. The fire is characterized using efficient features and detection of the same using a suitable processing. Every pixel is checked for the presence or absence of fire using color features, and periodic behavior in fire regions is also analyzed. In this paper we use combined approach of color detection, motion detection and area dispersion to detect fire in video data. Firstly, the algorithm locates desired color regions in video frames, and then determines the region in the video where there is any movement, and in the last step we calculate the pixel area of the frame. The combination of color, motion and area clues is used to detect fire in the video.
\end{abstract}

\section{Keywords}

RGB Color model, YCbCr color model, motion detection background subtraction, area dispersion.

\section{INTRODUCTION}

Automatic fire detection systems use physical sensors to detect and response of an fire. The physical sensor uses the chemical properties in the air are acquired by sensor and use by fire detection system to raise an alarm. This can also cause false alarms, The physical sensors are also not applicable for outdoor environment and in large infrastructure settings such as aircraft hangers, large tunnels. Due to the rapid development of digital camera technology and advanced content based image and video processing, there is a major trend to replace conventional fire detection system with computer vision based system.

There are lots of fire detection systems in which color is used in preprocessing step. Chen et al. [2], used RGB color channel information and create fire rules for the three channels separately. They used a moving detection algorithm in preprocessing step. To improve detection performance Toreyin et al. [3] proposed a real time algorithm for fire detection in video sequence. They used Combination of color and motion clues with fire flickers and then analyzed on wavelet domain to detect fire. Celik et al. [4], make use of fire detection rules after extracting foreground pixels by means of a change detection algorithm. They used method for the classification of fire/smoke pixels, separating them from the background.. Marbach et al. [5] propose a model where data are represented and analyzed in a YUV space. This model is used for representing the video data, where time derivation of luminance component $\mathrm{Y}$ used for first reduction of the candidate fire pixel, and chrominance component $\mathrm{U}$ and $\mathrm{V}$ used for classification whether or not the candidate pixels were in the fire section. In order to reduce false alarms, a simple motion detection algorithm is used to detect the areas containing the fire. Horng et al. [6] propose the HSI color space to roughly segment the fire-like region for brighter and darker environments.

Celik Turgay [7] proposes the CIE L*a*b color space for detection of fire pixels. In This color space the color tone are better grouped than in any other space.

In vision based fire detection system, there are three major features for fire: Color pixel, moving pixels and shape. The fire pixel can be classified as both in grayscale and color video sequences. Most of the fire detection system works on color video sequences.

It is assumed that the image capturing device produces its output in RGB color format, and these color information is used as a pre- processing step. During an occurrence of fire, smoke and flame can be seen. As the fire intensity is increases smoke will be visible.

A good color model selection for fire pixel detection and moving pixel detection play the critical role in computer visionbased fire detection system. The combination of color and motion clues used to detect the fire.

\section{MODEL FOR FIRE DETECTION}

In this section we will discuss the techniques proposed for fire detection.Fig. 1 shows the flowchart of proposed algorithm for fire detection in video.

In order to create the color model for fire we analyzed several images having fire. Since the color of fire is generally closer to red and has high illumination, and we can use this property to derive the required color model.

\subsection{RGB color model}

A fire image can be described by using its color properties. There are three different element of color pixel: R,G and B. The color pixel can be extracted into these three individual elements $\mathrm{R}, \mathrm{G}$ and $\mathrm{B}$, which is used for color detection.

RGB color model is used to detect red color information in image. In terms of RGB values, the corresponding inter-relation between $R, G$ and $B$ color channels: $R>G$ and $G>B$. The combined condition for the captured image can be written as: $R>G>B$. In fire color detection $R$ should be more stressed then the other component, and hence $\mathrm{R}$ becomes the domination color channel in an RGB image for fire.

This imposes the condition for $\mathrm{R}$ as to be over some predetermined threshold value $\mathrm{RTH}$.

All of these conditions for fire color in image are summarized as following:

Condition 1: $\mathrm{R}>\mathrm{RTH}$

Condition2: $\mathrm{R}>\mathrm{G}>\mathrm{B}$.

Where RTH is the Red color threshold value for fire. 


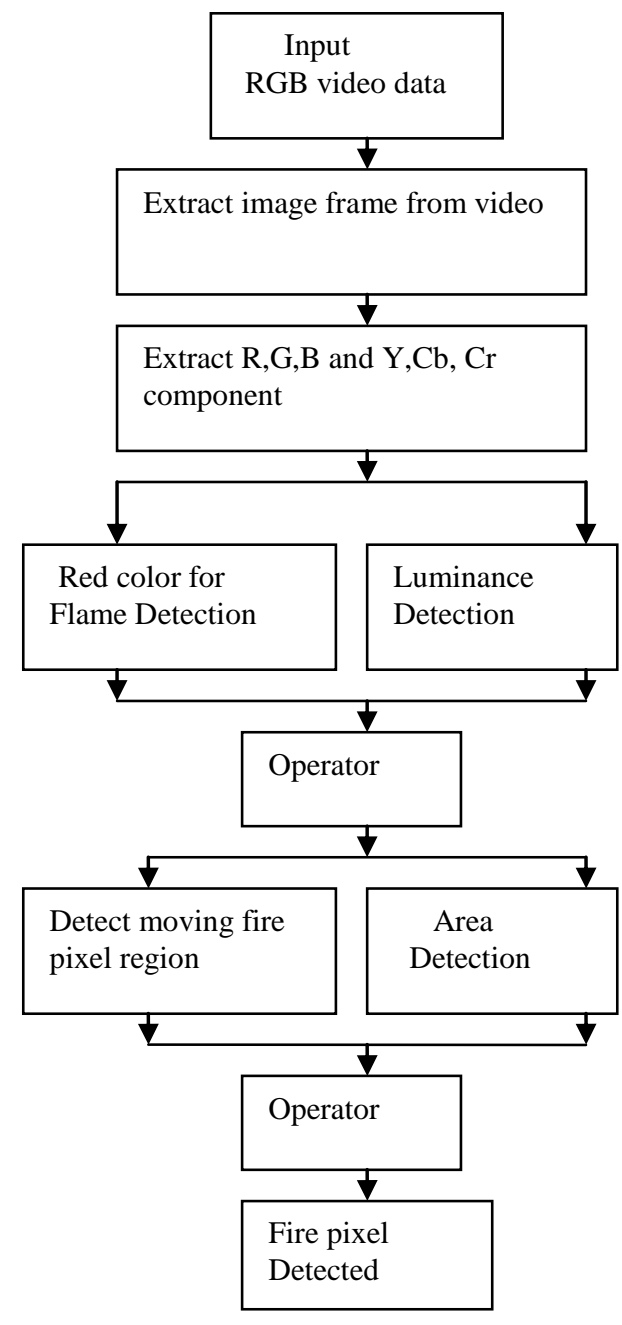

Fig.1. Flow chart of proposed algorithm for fire detection.

\subsection{YCbCr color model}

$\mathrm{YCbCr}$ color space is used in our model rather than other color spaces because of its ability to distinguish luminance information from chrominance information more effectively then other color model. In order to create $\mathrm{Y}, \mathrm{Cb}, \mathrm{Cr}$ components from obtained RGB Image. We will use color space transformation equation to transform each RGB pixel in corresponding $\mathrm{Y}$ Channel, $\mathrm{Cb}$ Channel, $\mathrm{Cr}$ Channel pixel to form a corresponding $\mathrm{Y}, \mathrm{Cb}, \mathrm{Cr}$ image.

When the image is converted from RGB to $\mathrm{YCbCr}$ color space, intensity and chrominance is easily discriminated.

$\mathrm{YCbCr}$ color space can be easily model as following for the fire:

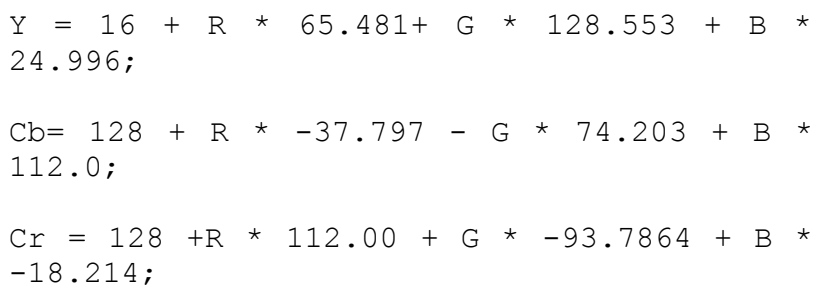

In $\mathrm{YCbCr}$ color space, $\mathrm{Y}^{\prime}$ is the luma component (the "black and white" or achromatic portion of the image) and $\mathrm{Cb}$ and $\mathrm{Cr}$ are the blue-difference and red-difference chrominance components, will be chosen intentionally because of its ability to separate illumination information from chrominance more effectively than the other color spaces. In $\mathrm{YCbCr}$ color space and analysis can be performed. For a fire pixel $\mathrm{Y}(\mathrm{x}, \mathrm{y})>=\mathrm{Cr}(\mathrm{x}$, y) $>=\mathrm{Cb}(\mathrm{x}, \mathrm{y})$, where a non-fire pixels don't satisfy this condition, where $(\mathrm{x}, \mathrm{y})$ is spatial location of a fire pixel. Such system can be useful for detecting forest fires where we can't put sensors at each location.

So we can summarize overall relation between $\mathrm{Y}(\mathrm{x}, \mathrm{y}), \mathrm{Cb}(\mathrm{x}, \mathrm{y})$ and $\operatorname{Cr}(\mathrm{x}, \mathrm{y})$ as follows:

$\mathrm{Y}(\mathrm{x}, \mathrm{y})>=\mathrm{Cr}(\mathrm{x}, \mathrm{y})>=\mathrm{Cb}(\mathrm{x}, \mathrm{y})$

Now, we can have some rules for fire detection:

$\begin{aligned} & \text { Rule1: } \mathrm{R} 1(\mathrm{x}, \mathrm{y}) \\ & \mathrm{B}(\mathrm{x}, \mathrm{y})))\end{aligned}$
$\quad 0$, otherwise

Rule2: $R 2(x, y)=1$, if $(R(x, y)>190) \& \&(G(x, y)>100) \& \&$ $(\mathrm{B}(\mathrm{x}, \mathrm{y})<140)$

0 , otherwise

Rule3: $\mathrm{R} 3(\mathrm{x}, \mathrm{y})=1$, if $\mathrm{Y}(\mathrm{x}, \mathrm{y})>=\mathrm{Cb}(\mathrm{x}, \mathrm{y})$

0 , otherwise

Rule4: $\mathrm{R} 4(\mathrm{x}, \mathrm{y})=1$, if $(\mathrm{Cr}(\mathrm{x}, \mathrm{y})>=\mathrm{Cb}(\mathrm{x}, \mathrm{y})$ 0 , otherwise

\subsection{Motion detection in video}

Identifying moving objects from a video sequence is a fundamental and critical task in many computer-vision applications. A common approach is to perform background subtraction, which is used to identify any moving objects from the portion of a video frame that differs significantly from a background model. There are basically three approaches used in background subtraction for motion detection in a continuous video stream. One of the most common approaches is to compare the current frame with the previous one or with something we call background. In this paper we use Frame differencing method to detect the moving pixels in image. Frame differencing uses the video frame at time $t-1$ as the background for the frame at time t. The binary background difference is generated by comparing the current frame with the background frame. The flowchart for motion detection is shown in Fig.2.

This method does have two major advantages. One obvious advantage is the modest computational load. Another is that the background model is highly adaptive. Since the background is based solely on the previous frame, it can adapt to changes in the background faster than any other method (at 1/fps to be precise). As we'll see later on, the frame difference method subtracts out extraneous background noise (such as waving trees), much better than the more complex approximate median and mixture of Gaussians methods.

For noise removal a Gaussian filter can be used but we needs to be cautious about blurring. 


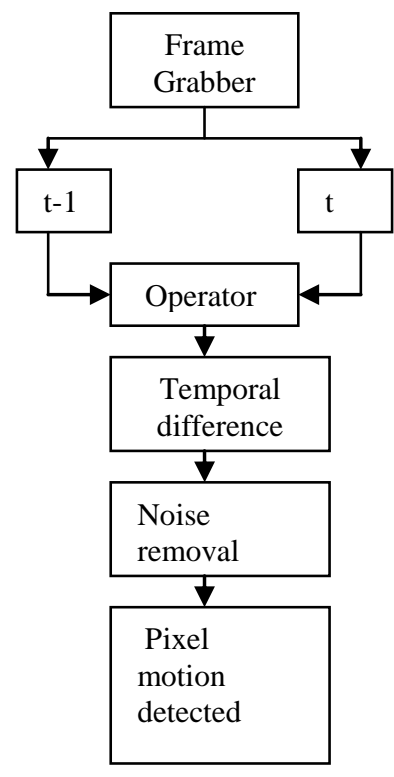

Fig 2. Flow chart for motion detection

\subsection{Area detection}

This method is used to detect dispersion of fire pixel area in the sequential frames. Area counts the number of pixels in an object [8]. In area detection method we take two sequential frames which comes out from color detector then we check dispersion in minimum and maximum coordinate of $\mathrm{X}$ and $\mathrm{Y}$ axis.

We can have model of area detection as in Fig.3.

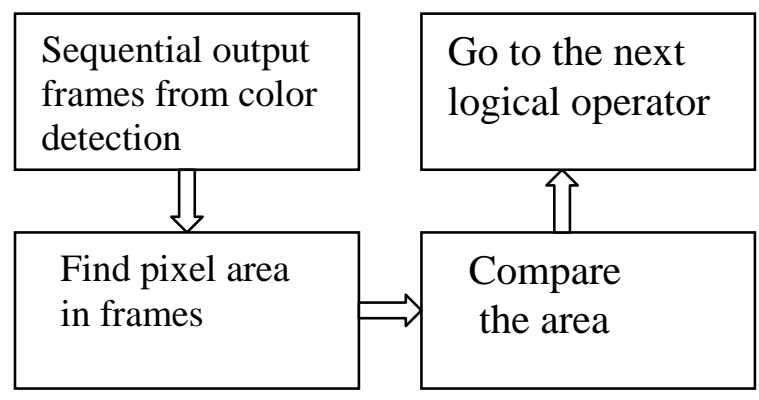

Fig.3 Model for area detection

\section{RESULTS}

We have taken two RGB image frames then algorithm is applied on it, and result is shown as in Fig.4(a) and Fig.4(b). Sample RGB image frames having fire, it contains sub images of different steps in algorithm: 1 st image frame, $2^{\text {nd }}$ image frame having flame, red component of fire pixel according to condition as mentioned above, motion is detected between these two frames, and last sub image shows the fire pixel detected in image.

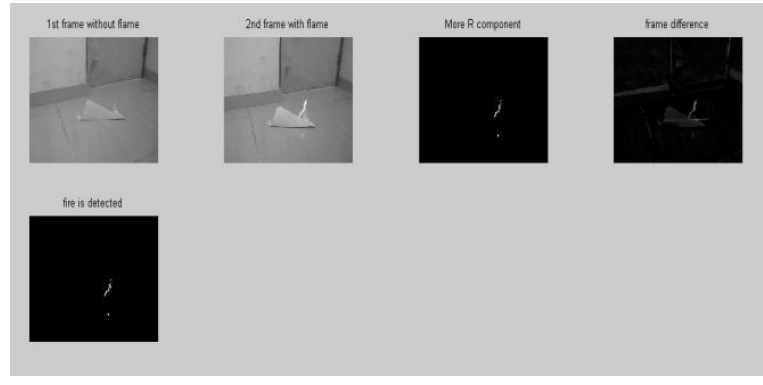

Fig. 4(a): First row shows the intermediate result of processing, and second row shows the final fire pixels

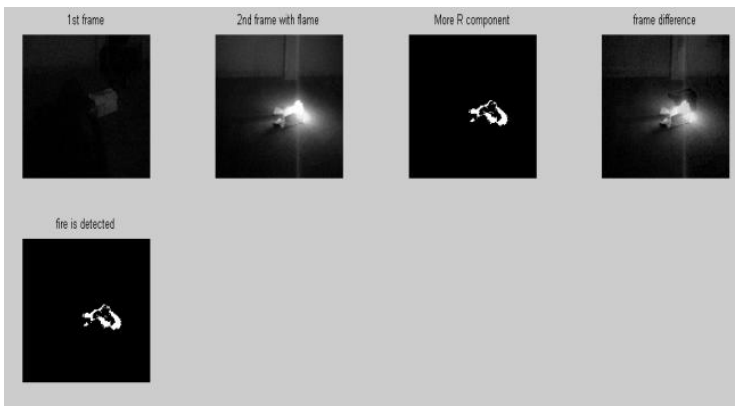

Fig. 4 (b): First row shows the intermediate result of processing, and second row shows the final fire pixels

Applications: Used to detect fire in forest, tunnel or any other unexpected fire.

\section{CONCLUSION}

In this paper an image based fire detection system was proposed, which is based on computer vision based techniques. We have collected a number of sequential frames from original video, which consists of fire and non fire images. The proposed method consists three main stages: - fire pixel detection using $\mathrm{RED}$ and $\mathrm{YCbCr}$ color model, moving pixel detection and analyzing shape of fire colored pixels in frames to detect fire pixel in image. The proposed method is applied on video sequences and then fire is detected.

Texture or shape information other then area can be used to improve system's fire detection performance. The performance of fire pixel can be further improved by applying smoke detection in the early stage of fire, along with the fire detection technique.

\section{REFERENCES}

[1] B.U. Toreyin, Y. Dedeoglu, and A.E. Cetin, "Computer Vision Based Method for Real-Time Fire and Flame Detection," Pattern Recognition Lett ., vol. 27, no. 1, 2006, pp. 49-58.

[2] Thou-Ho Chen, Ping-Hsueh Wu, and Yung-Chuen Chiou, "An early fire-detection method based on image processing," in Image Processing, 2004. ICIP '04. 2004 International Conference on, oct. 2004, vol. 3, pp. 1707 1710 Vol. 3.

[3] B.U. Toreyin, Y. Dedeoglu, and A.E. Cetin, "Flame detection in video using hidden markov models," Image Processing, 2005. ICIP 2005. IEEE International Conference on, sept. 2005, vol. 2, pp. II - 1230-3. 
[4] Turgay Celik, Hasan Demirel, Huseyin Ozkaramanli, and Mustafa Uyguroglu, "Fire detection using statistical color model in video sequences," J. Vis. Comun. Image Represent., vol. 18, pp. 176-185, April 2007.

[5] Giuseppe Marbach, Markus Loepfe, and Thomas Brupbacher," An image processing technique for fire detection in video images," Fire Safety Journal, vol. 41, no. 4, pp. $285-289,2006$.

[6] Wen-Bing Horng, Jian-Wen Peng, and Chih-Yuan Chen, "A new image-based real-time flame detection method using color analysis," Networking, Sensing and Control, 2005. Proceedings. 2005 IEEE, March 2005, pp. 100 105 .

[7] Turgay Celik, "Fast and Efficient Method for Fire Detection Using Image Processing," ETRI Journal, Volume 32, Number 6, December 2010.
[8] Dr. Nicolas Pronost," Inleiding Beeldverwerking Introduction to Image Processing", Computer Science Bachelor Program Utrecht University.

[9] W. Phillips III, M. Shah, and N. V. Lobo, "FlameRecognition in Video," Pattern Recognition Letters, v.23(1-3), pp.319-327, Jan. 2002.

[10] B.C. Ko, K.H. Chong, J.Y. Nam (2009): Fire Detection based on vision Sensor and Supportvector services Fire Safety Journal, vol. 44, pp. 322-329.

[11] Vipin V, " Image processing based fire detection ," International Journal of Emerging Technology and Advanced Engineering Website: www.ijetae.com (ISSN 2250-2459, Volume 2, Issue 2, February 2012).

[12] DongKeun Kim," Smoke Detection using Boundary Growing and Moments" , International Conference on Convergence and Hybrid Information Technology 2009. 